\title{
In situ magneto-optical ellipsometry data analysis for films growth control
}

\author{
O.A. Maximova ${ }^{a, b, c .}$, N.N. Kosyrev ${ }^{a, d}$, S.N. Varnakov ${ }^{a, b}$, S.A. Lyaschenko ${ }^{a, b}$, I.A. Yakovlev ${ }^{a, b}$, \\ I.A. Tarasov ${ }^{\text {a.b }}$, D.V. Shevtsov ${ }^{\text {a.b }}$, O.M. Maximova ${ }^{c}$, S.G. Ovchinnikov ${ }^{\text {a.b.e }}$ \\ 'Kirensky Institute of Physics, Federal Research Center KSC SB RAS, Krasnoyarsk 660036, Russia ' Reshetnev Siberian \\ State Aerospace University, Krasnoyarsk 660037, Russia 'Siberian Federal University, Krasnoyarsk 660041, Russia \\ Achinsk Branch of Krasnoyarsk State Agrarian University, Achinsk 662150, Russia
}

\section{ARTICLE INFO ABSTRACT}

Keywords: In this work we present the way of ferromagnetic films study by means of magneto-ellipsometry. The method of Magneto-optical Kerr effect interpretation of in situ magneto-optical ellipsometry spectra for real time growth control is described. The Ellipsometry method has been successfully tested on Si/SiO /Fe films within the model of a homogeneous semi-infinite

medium. As a result, the dielectric tensor components for Fe layer were calculated using a developed approach.

\section{Introduction}

In recent years, the magnetic materials for data storage and spintronic devices have deserved significant attention. There is a problem of an in situ real time control of nanomaterials synthesis [1] and their properties investigation because the in-air investigation of these structures is often impossible due to the high chemical activity of many materials used in this field. One of the best solutions of this problem is to use the optical and magneto-optical techniques. They are powerful, do not affect the sample and have some flexibility when being used in situ, directly in an ultrahigh vacuum chamber. We suggest that magneto-ellipsometry [2] meets all these requirements. This experimental technique combines ellipsometry [3] and the magneto-optical Kerr effect measurement within one setup with an ultra-high vacuum chamber and the electromagnet for magnetization reversal of the sample [4].

Although several attempts have been made to design a single setup for determining magneto-optical and conventional optical constants in it, few studies have focused on data processing that can be conducted right in the process of materials synthesis. Mok in his study [5] faced the necessity of carrying out an additional experiment. He had to determine magnetization in order to extract magnetic-field-dependent and independent parts in non-symmetric terms of a dielectric permittivity tensor. This indicates a need to write a sufficient data processing algorithm so that it is applicable to studying samples with magnetoellipsometry measurement without using any other setups despite that one that is used for synthesis. The use of this software would reduce the time and increase efficiency of experiment data analysis.
In this paper, we suggest a new approach to real time control of obtaining material parameters of magnetic thin films that can be applied right in the process of their growth by means of the in situ magneto-optical ellipsometry. In the end, to address the validity of the proposed method, we carried out an experiment on $\mathrm{Si}$ (substrate)/ $\mathrm{SiO}_{2}$ (layer)/Fe(layer) film study and compared the obtained values of material parameters with those in [6].

The results we present here indicate that the offered method is truly sufficient, simple, and reliable for in situ spectral magneto-ellipsometric measurements data interpretation.

\section{Magneto-ellipsometry data analysis}

Here we describe the method of interpretation of the magnetoellipsometric measurements data. We consider the case of electromagnetic wave incidence from non-magnetic dielectric medium (characterized by the refraction index $N_{0}=n_{0}-i k 0$ ) onto ferromagnetic metal (the refraction index $\mathrm{Nn}$ ) in the visible light range. $=-i k$

In the setup, a Cartesian coordinate system is defined with the $\mathrm{x}$-axis normal to the interfaces and pointing into the substrate from the sample surface. The $\mathrm{y}$-and $\mathrm{x}$-axis lie in the plane of incidence. There can be three configurations: longitudinal (L), transverse (T), and polar

(P) defined according to the direction of the magnetization vector. According to the design of the setup [4], we consider T-configuration in which magnetization is $\mathrm{z}$-axis directed, i.e perpendicular to the plane of incidence and parallel to the surface.

The key idea of the proposed approach is reported in [7], where it was applied toward the particular case of low magnetic field and 
consequently the use of small parameters. Here we consider a general case of experimental data processing for the model of a homogeneous semi-infinite medium without any constraints.

We suggest that magneto-ellipsometry technique gives an opportunity to determine all elements of the dielectric permittivity tensor $\varepsilon$ of the magnetized ferromagnetic metal [8]

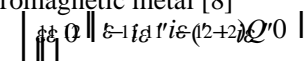

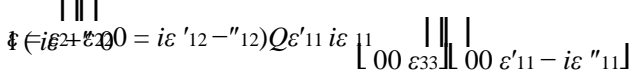

where $\varepsilon^{\prime}$ and $\varepsilon^{\prime \prime}$ are real and imaginary parts of medium permittivity, respectively, $Q=Q_{1}-i Q_{2}$ is a proportional to magnetization magneto-optical parameter. Diagonal tensor elements are responsible for refractive index and extinction coefficient, off-diagonal tensor elements are related to magnetooptical effects. So we get quite a lot of information on the sample if we know all the elements of dielectric permittivity tensor. The current work presents how to obtain the values of these elements from magneto-ellipsometric spectra without necessity of any additional ex situ measurements.

Let us denote the ellipsometric parameters in the non-magnetic condition as $\psi_{0}$ and $\Delta_{0}$. In the case of magneto-ellipsomertic characterization of the sample the surface transverse magneto-optical Kerr effect results in the ellipsometric angles corrections $\delta \psi$ and $\delta \Delta$. Thus, the ellipsometric parameters become $\psi 0+$ $\delta \psi, \Delta 0+\delta \Delta$. It means that we have four measured independent real-valued quantities $(\psi 0, \delta \psi, \Delta 0, \delta \Delta)$, as a result, we can derive four real-valued quantities $\left(\varepsilon^{\prime}, \varepsilon^{\prime \prime}, \varepsilon^{\prime}, \varepsilon^{\prime \prime} 12\right)$.

111112

There are four steps of data analysis:

$1 \quad$ Carrying out spectral ellipsometry $(\psi 0, \Delta 0)$ and magneto-optical Kerr effer measurements $(\psi 0+\delta \psi, \Delta 0+\delta \Delta)$.

2 Calculation of spectral dependences of refractive index (n) and extinction coefficient (k)

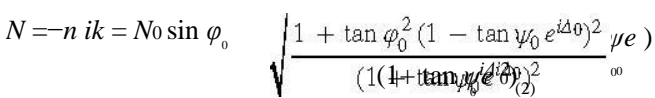

3. Theoretical calculation of the ellipsometric parameters $\psi 0, \delta \psi, \Delta 0, \delta \Delta$. Here we rewrite the basic equation of ellipsometry in the following way:

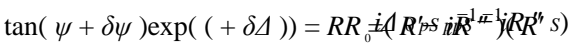

where $\mathrm{R}_{\mathrm{p}}$ and $\mathrm{R}_{\mathrm{s}}$ are complex reflection coefficients corresponding to in-plane p-polarization and out-of-plane s-polarization, respectively, real parts are marked by ', imaginary by ". According to mode conversion from the p-to the s-polarized channel we can write that

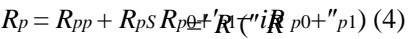

$R s=R S S+R S p=R s 0=R^{\prime} S 0-i R^{\prime \prime} s 0(5)$

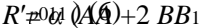

$R^{\prime \prime \# \# b l ~}(\not A \vec{B})-2 B A 1$

$R^{\prime}=1 \alpha^{22}((8 C-4 B C+4 A B D)$

$R^{\prime \prime}=22((\Phi D)-4 B D-4 A B C)$

$R^{\prime} ¥ 022(A C+2 B D)(10)$

$R^{\prime \prime} \operatorname{son}\left(2 B C 1+2 A D_{2}\right.$

where we have distinguished the magnetic field contribution and marked it by subscript 1 , non-magnetic summands - by subscript 0 . By substituting the determined values of $\mathrm{n}$ and $\mathrm{k}$ into Eqs. (6)-(11) and using the following notations

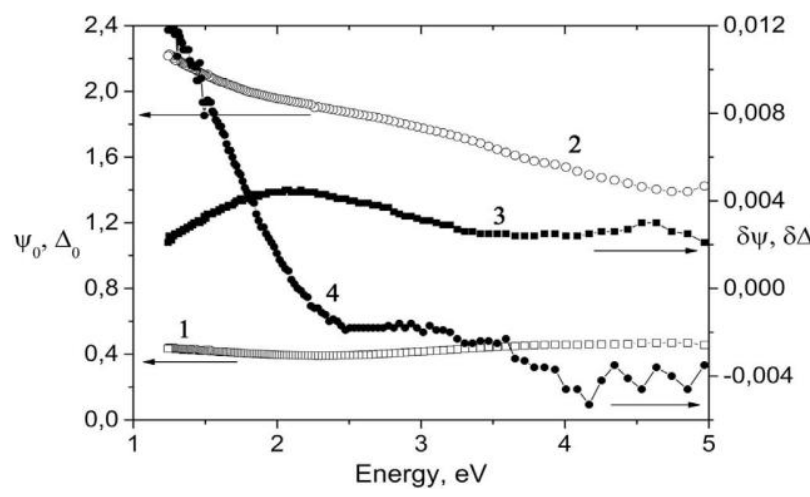

Fig. 1. The experimental and calculated values of ellipsometric parameters: $1-\psi 0,2-\Delta 0,3-\delta \psi, 4-$ $\delta \Delta$

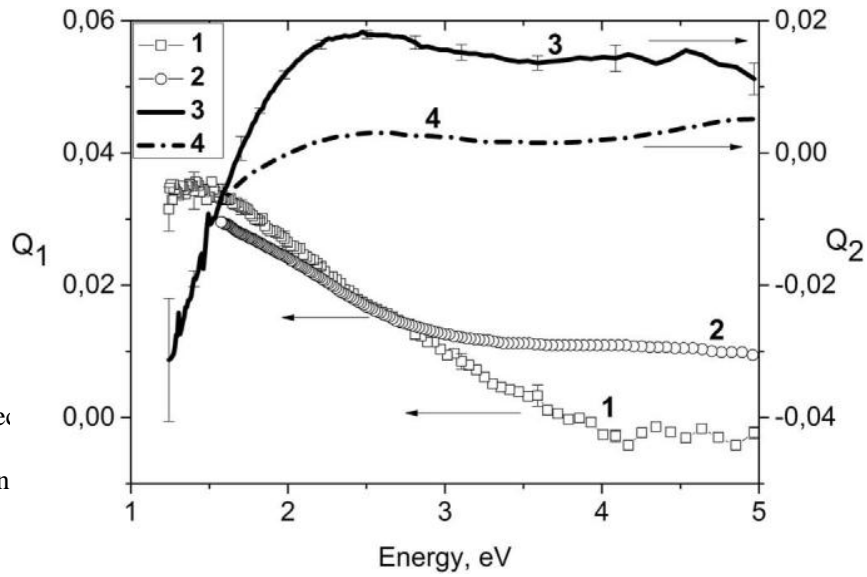

Fig. 2. Calculated values of real and imaginary parts of Fe magneto-optical coupling parameter $Q=Q 1$ $i Q 2$ in comparison with those obtained for $\mathrm{Fe}$ in [6]:1 - calculated values of $\mathrm{Q}_{1}, 2-\mathrm{Q}_{1}$ in [6],3 calculated values of $\mathrm{Q}_{2,4}-\mathrm{Q}_{2}$ in [6].

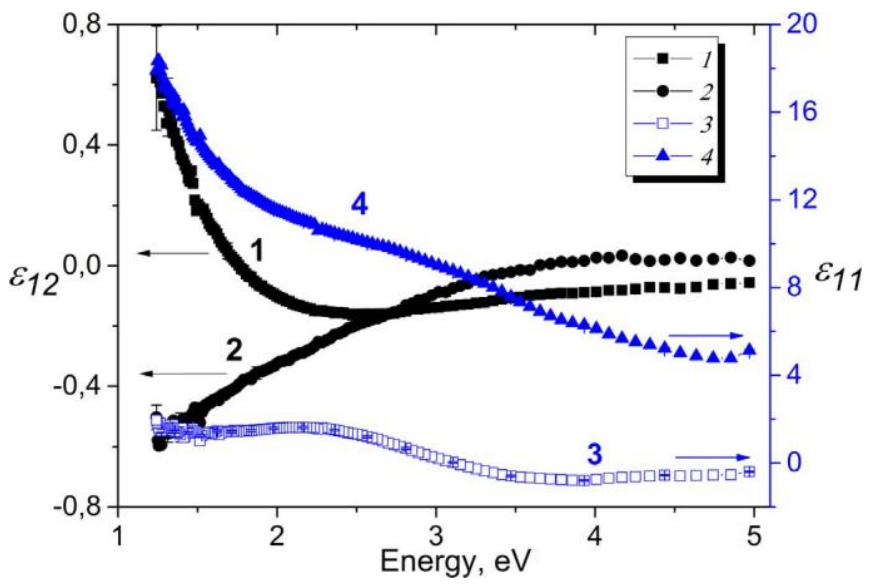

Fig. 3. The calculated values of real and imaginary parts of the Fe diagonal $\varepsilon 11$ and off-diagonal $\varepsilon_{12}$ dielectric permittivity tensor elements: $1-\varepsilon^{\prime \prime} 12,2-\varepsilon^{\prime} 12,3-\varepsilon^{\prime} 11,4-\varepsilon^{\prime \prime} 11$. 


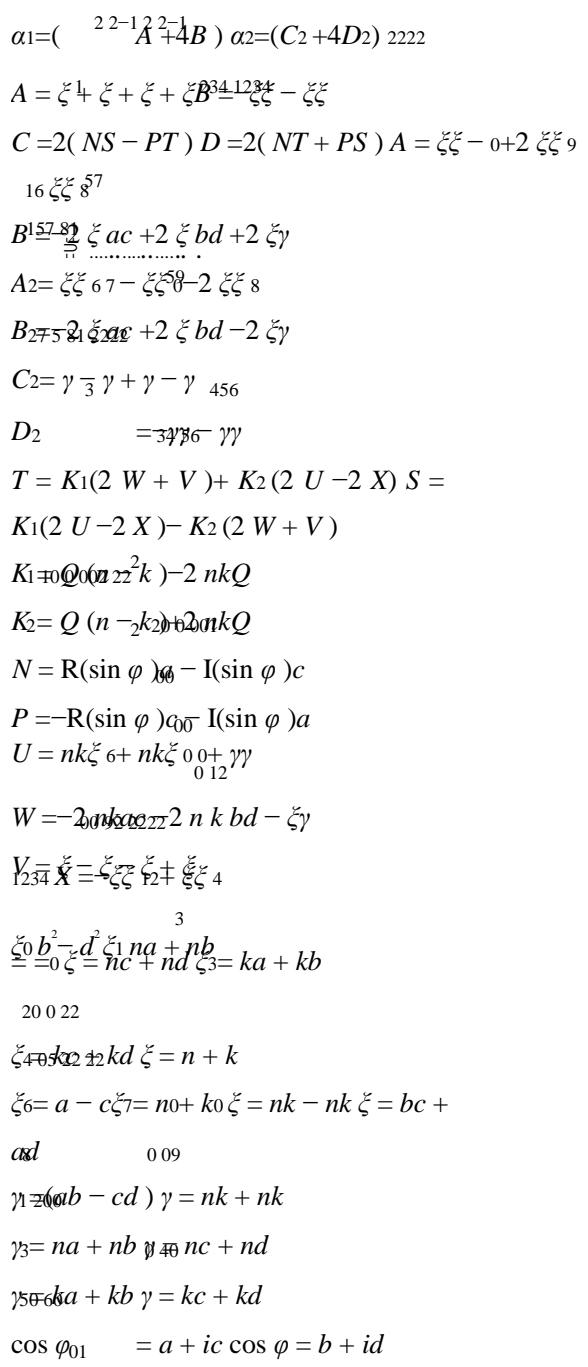

where $\varphi_{0}$ and $\varphi_{1}$ are the angles of incidence and refraction, respectively, while $\mathrm{a}, \mathrm{b}$ are real and $\mathrm{c}, \mathrm{d}$ are imaginary parts of $\cos \varphi_{0}$ and $\cos \varphi 1$, respectively, we obtain all necessary expressions for ellipsometric angles calculation.

4. Fitting to the experimental ellipsometric angles by the Nelder- Mead method [9]. As a result it yields the spectral dependences of real $\left(\mathrm{Q}_{1}\right)$ and imaginary $\left(\mathrm{Q}_{2}\right)$ parts of magneto-optical parameter $\mathrm{Q}$. Thus, we have information about all elements of the dielectric permittivity tensor.

\section{Results and discussion}

In order to demonstrate the method of interpretation of in situ magneto-ellipsometry measurements data, the sample in the form of polycrystalline $\mathrm{Fe}$ layer on the surface of $\mathrm{Si}(100) / \mathrm{SiO} 2$ was studied. The process of $\mathrm{SiO} / 2 \mathrm{Si}(100)$ substrate primary chemistry is specified in [10].

Fe film was made by ultrahigh vacuum thermal evaporation with deposition on the cool substrate, nevertheless the proposed data analysis approach can be also applied to data from the molecular beam epitaxy setups. Final thicknesses of $\mathrm{SiO}_{2}$ and $\mathrm{Fe}$ layers were

$3.84 \mathrm{~nm}$ and $160.5 \mathrm{~nm}$, respectively. During all the measurements the angle of incidence was fixed at $56^{\circ}$. While carrying out magneto-optical ellipsometry measurements the magnetization reversal of the sample was in the $\pm 2 \mathrm{kOe}$ field.

Then, the proposed algorithm for the interpretation of magnetoellipsometric measurement data for the model of a homogeneous semi-infinite medium was used. The values of refractive index, extinction coefficient and magneto-optical parameter $\mathrm{Q}$ of $\mathrm{Fe}$ layer in $\mathrm{Si} / \mathrm{SiO} / \mathrm{Fe}$ structure were obtained using the presented above approach. They were used for calculating $\psi 0, \delta \psi$, $\Delta 0, \delta \Delta$.In Fig. 1 the experimental and calculated values of these parameters are presented. Experimental data are shown by lines and calculated - by symbols. One can see that they fit each other. It is not surprising as the error of approximation by Nelder-Mead method was put to be 0.0001 .

Finally, we obtained magneto-optical coupling parameter $\mathrm{Q}$ values from these curves (Fig. 2), consequently we have completely determined all elements of the dielectric permittivity tensor (Fig. 3). The comparison of the $\mathrm{Fe}$ magneto-optical parameter $\mathrm{Q}$ with [6] shows a good qualitative agreement. Quantitatively, the curves are not similar as the thicknesses of $\mathrm{Fe}$ layer in the works differ: our sample $(\mathrm{Si} / \mathrm{SiO} / \mathrm{Fe})$ was $160.5 \mathrm{~nm}$ and the sample in [6] $(\mathrm{Si}(100 \mathrm{~g} / \mathrm{Fe})$ was $60 \mathrm{~nm}$ thick. In both cases the Fe thickness is greater than the optical skin depth so the results can be compared.

Thus, a new opportunity of in situ simultaneous characterization of optical and magneto-optical properties of films without carrying out any additional ex situ measurements has been demonstrated by means of magneto-ellipsometry. This approach can be used for thin films synthesis control.

\section{Acknowledgments}

The reported study was funded by Russian Foundation for Basic Research, Government of Krasnoyarsk Territory, Krasnoyarsk Region Science and Technology Support Fund to the research project Nos. 1642-243058 and 16-42-243060. The work was supported partly by the Russian Foundation for Basic Research, Grant nos. 16-32-00209 mol_a and 14-02-01211; the Complex program of SB RAS II.2P, project 0358-2015-0004; the Ministry of Education and Science of the RF (State task no. 16.663.2014); Grant Scientific School 7559.2016.2.

\section{References}

[1] D. Kumar, et al., J. Phys.: Conf. Ser 114 (2008) 012034

[2] G. Neuber, et al., Appl. Phys. Lett. 83 (2003) 4509.

[3] H. Fujiwara, Spectroscopic Ellipsometry Principles and Applications, John Wiley \& Sons, Chichester, 2007.

[4] S.V. Rykhlitskii, et al., Prib. Tekh. Eksp. 2 (2012) 165.

[5] K. Mok, et al., Phys. Rev. 84 (2011) 094413.

[6] G. Neuber, et al., Rev. Sci. Instrum. 76 (2005) 023910

[7] O.A. Maximova, et al., J. Struct. Chem. 55 (6) (2014) 1134-1141.

[8] A.V. Sokolov et al., Optical Properties of Metals, GIFML, Moscow, 1961.

[9] J.A. Nelder, A.D. Mead, Comput. J. 7 (1965) 308-313.

[10] N.V. Volkov, J. Appl. Phys. 109 (2011) 123924. 